\title{
Association between plasma ceramides and inducible myocardial ischemia in patients with established or suspected coronary artery disease undergoing myocardial perfusion scintigraphy
}

Alessandro Mantovani, $\mathrm{MD}^{1}$, Stefano Bonapace, $\mathrm{MD}^{2}$, Gianluigi Lunardi, $\mathrm{PhD}^{3}$, Matteo Salgarello, MD ${ }^{4}$, Clementina Dugo, MD² , Guido Canali, MD², Christopher D. Byrne, MB $\mathrm{BCh}, \mathrm{PhD}^{5,6}$, Stefania Gori, MD${ }^{3}$, Enrico Barbieri, $\mathrm{MD}^{2}$, Giovanni Targher, $\mathrm{MD}^{1}$

${ }^{1}$ Section of Endocrinology, Diabetes and Metabolism, Department of Medicine, University and Azienda Ospedaliera Universitaria Integrata of Verona, Verona, Italy

2Division of Cardiology, "Sacro Cuore-Don Calabria" Hospital, Negrar (VR), Italy

${ }^{3}$ Division of Medical Oncology, "Sacro Cuore-Don Calabria" Hospital, Negrar (VR), Italy

${ }^{4}$ Division of Nuclear Medicine, "Sacro Cuore-Don Calabria" Hospital, Negrar (VR), Italy

${ }^{5}$ Nutrition and Metabolism, Faculty of Medicine, University of Southampton, UK

${ }^{6}$ Southampton National Institute for Health Research Biomedical Research Centre, University Hospital Southampton, Southampton General Hospital, Southampton SO16 6YD, UK

Short title: Plasma ceramides and inducible myocardial ischemia

Word count: abstract 259; text 3,861 (excluding title page, abstract, abbreviation list, acknowledgments and references); n. 5 Tables + n. 1 Figure (+ n. 2 Supplementary Tables; n. 1 Supplementary Figure). References $=26$

Address of correspondence:

Prof. Giovanni Targher

Section of Endocrinology, Diabetes and Metabolism

University and Azienda Ospedaliera Universitaria Integrata

Piazzale Stefani, 1

37126 Verona, Italy

Tel: 0039/045-8123748

Fax: 0039/045-8027314

E-mail: giovanni.targher@univr.it 


\section{ABSTRACT}

Background: Recent studies have suggested that specific plasma ceramides are independently associated with major adverse cardiovascular events in patients with coronary artery disease (CAD), but it is currently unknown whether plasma ceramide levels are associated with stress-induced reversible myocardial ischemia.

Methods: We measured six previously identified high-risk plasma ceramide molecules [Cer(d18:1/16:0), Cer(d18:1/18:0), Cer(d18:1/20:0), Cer(d18:1/22:0), Cer(d18:1/24:0), and Cer(d18:1/24:1)] in 167 consecutive patients with established or suspected CAD who underwent either exercise or dypiridamole myocardial perfusion scintigraphy (MPS) for various clinical indications. Plasma ceramide levels were measured by a targeted liquid chromatography-tandem mass spectrometry assay both at baseline and after MPS.

Results: Seventy-eight patients had inducible myocardial ischemia on stress MPS. Women had significantly higher circulating levels of basal and post-stress Cer(d18:1/16:0) and Cer(d18:1/18:0) compared to men, whereas all other plasma ceramides did not differ between the sexes. Of the six measured plasma ceramides, basal Cer(d18:1/24:1) showed the strongest association with the presence of stress-induced myocardial perfusion defects in univariate analysis (unadjusted-odds ratio 1.48 per 1-SD increment, 95\% confidence interval 1.08-2.04). Notably, after adjustment for age, sex, smoking, dyslipidemia, hypertension, diabetes, prior history of CAD, left ventricular ejection fraction, and type of stress testing (exercise vs. dypiridamole), all measured ceramides, except for plasma Cer(d18:1/24:0), were independently associated with the presence of inducible myocardial ischemia.

Conclusions: Distinct plasma ceramides are positive and independent predictors of stress-induced myocardial perfusion defects in patients with established or suspected CAD referred for clinically indicated MPS. Further research is needed to examine whether distinct plasma ceramides could be a useful therapeutic target for treatment and management of CAD.

Key words: ceramides, coronary artery disease, cardiovascular risk, myocardial perfusion scintigraphy 


\section{LIST OF ABBREVIATIONS USED}

CAD, coronary artery disease

Cer, ceramide

e-GFR, glomerular filtration rate

$\mathrm{HbA1c}$, hemoglobin A1c

MPS, myocardial perfusion scintigraphy

SPECT, single photon emission computed tomography

99mTc-MIBI, Technetium-99m 2-methoxy-isobutyl-isonitrile 


\section{INTRODUCTION}

Ceramides are a family of complex lipid molecules composed of sphingosine and a fatty acid, and are found in high concentrations in cell membranes [1,2]. In addition to their structural role in the physicochemical properties of cell membranes, ceramides play a key role in cellular stress response, inflammation, apoptosis and various signaling pathways $[1,2]$. Literature on ceramide biology also links these molecules with many atherosclerotic processes, such as lipoprotein aggregation, uptake of lipoproteins and accumulation of cholesterol within macrophages, regulation of nitric oxide synthesis, production of reactive oxygen species, platelet activation, and expression of various cytokines. Moreover, experimental studies in animals have also shown that ceramides are associated with the development of atherosclerosis [3-5].

In recent years, large prospective studies have identified three specific plasma ceramides [Cer(d18:1/16:0), Cer(d18:1/18:0) and Cer(d18:1/24:1] as significant predictors of adverse cardiovascular outcomes in patients with established coronary artery disease (CAD) or clinical suspicion for acute coronary syndromes and in apparently healthy individuals, over and above currently used lipid markers and other traditional cardiovascular risk factors [69].

To our knowledge, there is currently a lack of information about the association between these previously identified high-risk plasma ceramides and the presence of stress-induced reversible myocardial ischemia on myocardial perfusion scintigraphy (MPS), i.e., a valuable prognostic test in CAD [10], among patients with established or suspected CAD. We believe that this topic is clinically relevant as it could expand our knowledge(s) about the complex mechanisms underlying the development of CAD, and could also provide new insights into the prevention and management of CAD in this particularly high-risk patient population.

Thus, the aim of this cross-sectional study was to explore whether distinct plasma ceramide species were significantly associated with the presence of inducible myocardial ischemia in a sample of consecutive patients with established or suspected CAD undergoing stress MPS. 


\section{MATERIAL AND METHODS}

\subsection{Patients}

We examined 167 patients with established or suspected CAD, attending the Cardiology outpatient service of the "Sacro Cuore" Hospital of Negrar (VR), who consecutively performed a stress MPS for various clinical indications (e.g., chest pain, palpitations, dyspnea, suspected ischemic electrocardiographic alterations, echocardiographic abnormalities or presence of multiple cardiovascular risk factors) over a period of four months (from April to July 2017). Of these patients, 94 patients performed an exercise stress testing with bicycle ergometry, whereas 73 patients performed a pharmacological stress testing with dipyridamole infusion. The pharmacological stress testing was used for patients who were unable to perform the standard exercise stress test.

We excluded from the study patients with: (i) a documented history of malignancy, cirrhosis and kidney failure (defined as either estimated glomerular filtration rate $<15$ $\mathrm{ml} / \mathrm{min} / 1.73 \mathrm{~m}^{2}$ or chronic dialysis); (ii) a documented history of overt hyperthyroidism or hypothyroidism; and (iii) known active infectious diseases (including myocarditis and endocarditis).

The local Ethics Committee approved the study protocol. All participants gave their written informed consent for participation in this research.

\subsection{Clinical and laboratory data}

Body mass index was measured as kilograms divided by the square of height in meters. Blood pressure was measured with a standard sphygmomanometer after patient had been seated quietly for at least $5 \mathrm{~min}$. Patients were considered to have arterial hypertension if their blood pressure was $\geq 140 / 90 \mathrm{mmHg}$ or if they were taking any anti-hypertensive agents. Information on smoking status and use of medications was obtained from all patients via interviews during medical examinations.

Venous blood samples were drawn in the morning after an overnight fast. Measurements of the plasma levels of lipids, creatinine (measured using a compensated rate-blanked Jaffè kinetic assay) and other biochemical blood parameters were obtained using standard laboratory procedures at the central Laboratory of the hospital for all patients. Plasma LDL-cholesterol was calculated using the Friedewald's equation. The glomerular filtration 
rate (eGFR $\mathrm{MDRD}_{\mathrm{M}}$ ) was estimated using the four-variable Modification of Diet in Renal Disease (MDRD) study equation [11]. In patients with established diabetes hemoglobin A1c (HbA1c) was measured using the BioRad HPLC analyser.

Pre-existing history of CAD was defined as a documented history of myocardial infarction, angina pectoris or coronary revascularization procedures. The diagnosis of permanent atrial fibrillation was made on the basis of medical history (from reviewing hospital and physician charts from all patients) and standard 12-lead electrocardiograms. Pre-existing history of valvular heart diseases was confirmed by reviewing medical records of the hospital and echocardiograms. Chronic kidney disease was defined as the presence of eGFR $_{M D R D}<60 \mathrm{~mL} / \mathrm{min} / 1.73 \mathrm{~m}^{2}$ [11]. The presence of chronic obstructive pulmonary disease was confirmed by reviewing medical records of the hospital, including results of lung function tests. Dyslipidemia was defined as a total cholesterol level $\geq 5.2 \mathrm{mmol} / \mathrm{L}$ and/or use of any lipid-lowering drugs. Previously known diabetes was defined as selfreported physician-diagnosed diabetes, or use of hypoglycemic medications (insulin or oral agents).

\subsection{Plasma ceramide measurements}

Blood samples for plasma ceramide measurements were obtained in ethylenediamine tetra-acetic acid (EDTA)-containing tubes both immediately before and after stress MPS. Plasma was collected within two hours from withdrawal and stored at $-80^{\circ} \mathrm{C}$ until analysis. Ceramides were purchased from Avanti Polar Lipids Inc. (Alabaster, Alabama, USA). Plasma concentrations of N-palmitoyl-D-erythro-sphingosine [Cer(d18:1/16:0)], N-stearoylD-erythro-sphingosine [Cer(d18:1/18:0)], N-arachidoyl-D-erythro-sphingosine [Cer(d18:1/20:0], N-behenoyl-D-erythro-sphingosine [Cer(d18:1/22:0)], N-lignoceroyl-Derythro-sphingosine [Cer(d18:1/24:0)], N-nervonoyl-D-erythro-sphingosine [Cer(d18:1/24:1)] were measured both before and after stress MPS by liquid-liquid extraction with 2-propanol:ethyl acetate $(4: 1 \mathrm{~V} / \mathrm{v})$ and gradient reverse phase chromatography on an Agilent Poroshell $120 \mathrm{C} 18$ column $(4.6 \times 50 \mathrm{~mm}, 2.7 \mu \mathrm{m})$ [12]. Nheptadecanoyl-D-erythro-sphingosine [Cer(d18:1/17:0)] was used as internal standard. The apparatus consisted of an Agilent 1290 UHPLC system coupled with an Agilent 6495 Triple Quadrupole LC/MS system. Mobile phases consisted in LC-MS grade water (A), acetonitrile with $0.1 \%$ formic acid (B) and $10 \mathrm{mM}$ ammonium acetate in 2-propanol (C). $[\mathrm{M}+\mathrm{H}]^{+} \rightarrow 264$ MRM transition was selected to quantify each ceramide. Calibration 
standards (six points) were daily prepared in surrogate matrix ( $5 \%$ bovine serum albumin) at concentration range from 1.0 to $0.031 \mu \mathrm{M} / \mathrm{L}$ for $\operatorname{Cer}(\mathrm{d} 18: 1 / 16: 0), \operatorname{Cer}(\mathrm{d} 18: 1 / 18: 0)$ and $\operatorname{Cer}(d 18: 1 / 20: 0)$, and from 10 to $0.31 \mu \mathrm{M} / L$ for $\operatorname{Cer}(d 18: 1 / 22: 0), \operatorname{Cer}(d 18: 1 / 24: 0)$ and Cer(d18:1/24:1), respectively. Linearity regression coefficient was $R^{2}>0.99$ for all ceramides. Inter-assay and intra-assay coefficients of variations for precision and accuracy were less than $15 \%$. No matrix interference or carryover was observed. All measurements of plasma ceramides were performed by an expert laboratory technician, who was blinded to the basal and post-stress statuses of ceramide sampling or any other clinical details of participants.

\subsection{Myocardial perfusion scintigraphy}

Rest and stress studies were done in all patients in a two-day $(2 \times 370 \mathrm{MBq})$ protocol using technetium-99m 2-methoxy-isobutyl-isonitrile (99mTc-MIBI), according to local standardized clinical routine [13]. Stress testing was performed with bicycle exercise in 94 $(56.3 \%)$ patients and with dipyridamole infusion in $73(43.7 \%)$ patients, respectively. During the bicycle exercise test, $99 \mathrm{mT}$ T-MIBI was injected at peak symptom-limited exercise testing. Bicycle exercise was performed following the Bruce protocol; electrocardiogram and blood pressure were recorded at rest and every two minutes during exercise and recovery. $370 \mathrm{MBq}$ of $99 \mathrm{mTc}-\mathrm{MIBI}$ was injected at rest and at peak exercise. Images were acquired $60 \mathrm{~min}$ after the injection of radiopharmaceutical drug on two phases (at baseline and after bicycle ergometry test). The single photon emission computed tomography (SPECT) acquisition protocol usually started with the resting part, followed by SPECT at stress the day after. SPECT was performed using a double-head gamma-camera (Millennium MG, GE) equipped with two scintillation detectors, angled $90^{\circ}$, with high resolution and low energy parallel-hole collimators. The protocol included 32 projections, 40-second projections, and 12 frames per cycle used in association with a $15 \%$ window centred on the $140-\mathrm{keV}$ photo peak of $99 \mathrm{mTc}-\mathrm{MIBI}$. An expert nuclear medicine physician, who was blinded to the participants' details, including ceramide measurements, classified the amount of inducible myocardial ischemia according to the number of stress-induced myocardial segments affected [10]. Left ventricular ejection fraction, end-diastolic and end-systolic volumes were also automatically calculated in all patients, using quantitative analysis of gated myocardial perfusion SPECT images. A similar protocol was used when myocardial perfusion scintigraphy was performed using a 
pharmacologic stress with intravenous dipyridamole (at a dose of $0.56 \mathrm{mg} / \mathrm{kg}$ over 4 minutes) in those patients, who were unable to exercise or who exercised sub-maximally.

\subsection{Statistical analysis}

Data are reported as means $\pm S D$ or percentages. Differences in main clinical and biochemical characteristics of patients stratified by presence or absence of stress-induced reversible myocardial perfusion defects on MPS were assessed using either the unpaired Student's $t$-test (for normally distributed variables) or the Mann-Whitney $U$ test (for nonnormally distributed variables, i.e., plasma ceramides, triglycerides and e-GFR $\left.R_{M D R D}\right)$. The chi-squared test was used to test for between-group differences among the categorical variables. Multivariate logistic regression analysis was used to evaluate the independent association between plasma ceramides (included as a continuous measure, i.e., for each SD increment) and the presence of stress-induced myocardial perfusion defects on MPS after adjustment for established cardiovascular risk factors. We performed three forcedentry logistic regression models: the $1^{\text {st }}$ model was unadjusted (unadjusted model); the $2^{\text {nd }}$ model was adjusted for age and sex (adjusted model 1 ); and, finally, the $3^{\text {rd }}$ model was additionally adjusted for smoking, dyslipidemia, hypertension, diabetes, prior history of CAD, left ventricular ejection fraction, and type of stress testing (exercise vs. dipyridamole) (adjusted model 2). Covariates included in these multivariate regression models were selected as potential confounding factors based on their significance in univariate analyses or based on their biological plausibility. A $p$-value $<0.05$ was considered to be statistically significant. Statistical analyses were performed using the STATA $₫$ software, version 14.2 (STATA, College Station, Tx, USA).

\section{RESULTS}

Overall, 167 (128 men and 39 women) patients with established or suspected CAD consecutively underwent stress MPS for various clinical indications; their mean age was 70 years; $56 \%$ had a prior history of CAD, $80 \%$ had hypertension, $>90 \%$ had dyslipidemia and $24 \%$ had established type 2 diabetes. A total of 78 patients had stress-induced reversible myocardial perfusion defects on MPS. 
The main clinical and biochemical characteristics of patients stratified by presence or absence of stress-induced reversible myocardial perfusion defects are summarized in Table 1. Compared with those without any myocardial perfusion defect, patients with inducible myocardial perfusion defects were more likely to be male, older, hypertensive and had a greater prevalence of established CAD. They also had a lower left ventricular ejection fraction (quantified by gated myocardial perfusion SPECT) and were more frequently treated with antiplatelet agents, anticoagulants, beta-blockers, nitrates or statins. Notably, among the different types of statins used by the treated patients $(n=104$; $62 \%$ of total), atorvastatin was the most frequently used $(73 \%)$, followed by simvastatin $(22 \%)$ and rosuvastatin (5\%); no patients were treated with pravastatin or fluvastatin. Conversely, body mass index, dyslipidemia, smoking, diabetes, chronic kidney disease and other comorbid conditions as well as the current use of hypoglycemic agents, reninangiotensin system inhibitors, diuretics, calcium-channel blockers, digitalis or antiarrhythmic drugs did not significantly differ between the two groups of patients.

The main clinical and biochemical characteristics of patients stratified by type of stress on MPS (exercise vs. dipyridamole) are shown in Table 2. No significant differences were observed in many clinical/biochemical variables and prevalence of stress-induced reversible myocardial perfusion defects between patients undergoing either exercise or dipyridamole MPS. The two groups of patients differed only for age and prevalence of hypertension and diabetes that were significantly higher in the dipyridamole group than in the exercise group, whereas LV ejection fraction was (slightly) lower in the dipyridamole group. These differences possibly reflect the clinical indications of these two stress tests in clinical practice.

Table 3 shows the basal and post-stress levels of plasma ceramides in patients stratified by presence or absence of stress-induced reversible myocardial perfusion defects on MPS. After adjusting for age and sex, patients with inducible myocardial ischemia had significantly higher levels of both basal and post-stress $\operatorname{Cer}(\mathrm{d} 18: 1 / 24: 1)$, as well as their ratios to $\operatorname{Cer}(\mathrm{d} 18: 1 / 24: 0)$ compared to those without any myocardial perfusion defect. In addition, the former also had higher basal plasma Cer(d18:1/20:0), Cer(d18:1/22:0) and $\operatorname{Cer}(d 18: 1 / 24: 1)$ concentrations. Conversely, the levels of plasma $\operatorname{Cer}(\mathrm{d} 18: 1 / 16: 0)$, Cer(d18:1/18:0) and Cer(d18:1/24:0), both at baseline and after stress, did not significantly differ between the two groups. 
As also shown in the table, there were mainly marginal differences in plasma ceramide levels before and after stress MPS. Figure 1 shows the percentages of patients responding with an increase (arbitrarily defined as an increment $\geq 10 \%$ over the basal), decrease ( $\geq-10 \%$ of basal) or no changes (from $0 \%$ to $+/-9.9 \%$ of basal) in the measured plasma ceramide levels after stratification by presence or absence of stress-induced reversible myocardial perfusion defects on MPS.

In Supplementary Table 1 are reported the plasma levels of basal and post-stress ceramides in patients grouped by sex. Women had significantly higher circulating levels of basal and post-stress $\operatorname{Cer}(\mathrm{d} 18: 1 / 16: 0)$ and $\operatorname{Cer}(\mathrm{d} 18: 1 / 18: 0)$ compared to men. Conversely, the levels of Cer(d18:1/20:0), Cer(d18:1/22:0), Cer(d18:1/24:0) and Cer(d18:1/24:1), as well as their ratios to $\operatorname{Cer}(\mathrm{d} 18: 1 / 24: 0)$, both at baseline and after stress, were not significantly different between the two sexes.

Supplementary Table 2 shows the plasma levels of basal and post-stress ceramides in patients stratified by type of stress on MPS (exercise vs. dipyridamole). Compared to the exercise group, patients belonging to the dipyridamole group had higher basal and poststress levels of Cer(d18:1/16:0), Cer(d18:1/18:0) and Cer(d18:1/24:1) as well as higher basal and post-stress ratios of Cer(d18:1/16:0)/Cer(d18:1/24:0), Cer(d18:1/18:0)/Cer(d18:1/24:0) and Cer(d18:1/24:1)/Cer(d18:1/24:0). Within each patient group, most of the post-stress ceramide levels increased more markedly in the exercise group compared to the dipyridamole group.

Table 4 shows the association of basal plasma ceramides and their ratios to Cer(d18:1/24:0) (for each SD increment) with the presence of inducible myocardial ischemia after stress MPS. In univariate analyses (unadjusted models), only baseline Cer(d18:1/24:1) was significantly associated with the presence of stress-induced reversible myocardial perfusion defects (unadjusted-odds ratio 1.48, 95\% Cl 1.08-2.04), whereas all other plasma ceramides were not. However, it should be noted that the association between most of the measured plasma ceramides and the presence of stressinduced reversible myocardial perfusion defects became statistically significant after adjustment for age, sex (model 1) and other traditional cardiovascular risk factors (fully adjusted model 2 ). Only baseline plasma $\operatorname{Cer(d18:1/24:0)~levels~did~not~significantly~}$ 
correlate with inducible myocardial ischemia neither in univariate analysis nor in multivariate regression analysis. With regard to the ratios of each ceramide to Cer(d18:1/24:0), we found that Cer(d18:1/20:0)/Cer(d18:1/24:0), Cer(d18:1/22:0)/Cer(d18:1/24:0) and Cer(d18:1/24:1)/Cer(d18:1/24:0) were independently associated with the presence of inducible myocardial ischemia after adjusting for traditional cardiovascular risk factors (regression model 2). Notably, in all aforementioned multivariate regression models male sex, lower left ventricular ejection fraction and prior history of CAD were the only other risk factors that were independently associated with the presence of inducible myocardial ischemia, whereas any of the components for dyslipidemia (total cholesterol, LDL-cholesterol, triglycerides) were not.

As shown in Table 5, almost identical results were observed even when the aforementioned logistic regression models were repeated including post-stress plasma ceramides [and their ratios to $\operatorname{Cer}(\mathrm{d} 18: 1 / 24: 0)$ ]. Also in this case, only post-stress Cer(d18:1/24:1) was significantly associated with the presence of stress-induced reversible myocardial perfusion defects in univariate analysis, whereas all other plasma ceramides were not. Again, the association between the plasma levels of most post-stress ceramides - except for plasma Cer(d18:1/24:0) - and the presence of stress-induced myocardial perfusion defects became significant after adjustment for traditional risk factors and other potential confounding variables (fully adjusted model 2 ). With regard to the ratios of each ceramide to $\operatorname{Cer}(\mathrm{d} 18: 1 / 24: 0)$, we found that only Cer(d18:1/22:0)/Cer(d18:1/24:0) and Cer(d18:1/24:1)/Cer(d18:1/24:0) were independently associated with the presence of inducible myocardial ischemia (model 2). Again, in all aforementioned multivariate regression models male sex, lower left ventricular ejection fraction and prior history of CAD were the only other known risk factors that were independently associated with the presence of inducible myocardial ischemia.

Similar results were observed even when the aforementioned logistic regression analyses were separately performed in those with $(n=94)$ and those without $(n=73)$ a prior history of $\mathrm{CAD}$, although the strength of the associations appeared to be greater in those without a history of CAD at baseline (data not shown). 


\section{DISCUSSION}

The novel results of this exploratory study provide evidence that previously identified highrisk plasma ceramide molecules are independently associated with inducible myocardial ischemia in a sample of consecutive patients with history of or clinical suspicion for CAD referred for clinically indicated stress MPS (i.e., an accurate imaging technique for the early diagnosis and quantification of CAD) [10]. In particular, higher levels of plasma $\operatorname{Cer}(d 18: 1 / 16: 0), \operatorname{Cer}(d 18: 1 / 18: 0), \operatorname{Cer}(d 18: 1 / 20: 0), \operatorname{Cer}(d 18: 1 / 22: 0)$ and $\operatorname{Cer}(d 18: 1 / 24: 1)$, both at baseline and after stress MPS, were significantly associated with the presence of inducible myocardial ischemia after adjustment for traditional cardiovascular risk factors, prior history of CAD, left ventricular ejection fraction, and type of myocardial stress testing (exercise vs. dipyridamole). Conversely, plasma Cer(d18:1/24:0) levels did not significantly associate with inducible myocardial ischemia in this high-risk patient group. Notably, these plasma ceramides were associated with inducible myocardial ischemia also in those patients, who were already treated with statins (most of whom achieving the target LDLcholesterol levels), and could represent, therefore, a potential marker of coronary residual risk. In addition, we also found that compared to the exercise group, patients of the dipyridamole group had significantly higher basal and post-stress levels of

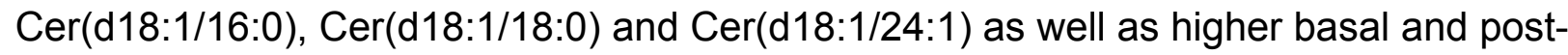
stress ratios of $\operatorname{Cer}(\mathrm{d} 18: 1 / 16: 0) / \operatorname{Cer}(\mathrm{d} 18: 1 / 24: 0), \operatorname{Cer}(\mathrm{d} 18: 1 / 18: 0) / \operatorname{Cer}(\mathrm{d} 18: 1 / 24: 0)$ and $\operatorname{Cer}(\mathrm{d} 18: 1 / 24: 1) / \operatorname{Cer}(\mathrm{d} 18: 1 / 24: 0)$. Within each patient group, most of the post-stress ceramide levels increased more markedly in the exercise group compared to the dipyridamole group. Finally, women had significantly higher levels of basal and post-stress Cer(d18:1/16:0) and Cer(d18:1/18:0) than men, whereas all other measured plasma ceramides were not significantly different between men and women.

Recognition of "high-risk" molecular lipids, beyond plasma LDL-cholesterol levels, could further improve our knowledge(s) about the complex mechanisms potentially involved in the development and progression of coronary atherosclerosis, and could be also useful for establishing an individual's cardiovascular risk or determining whether a residual risk remains [14].

Over the last years, accumulating experimental and clinical evidence has provided new insights into the potential role of distinct ceramide species in the development and 
progression of CAD [6-9,15-18]. Synthesis of ceramides from saturated fats and sphingosine occurs in all tissues, mainly through the actions of at least six identified tissuespecific ceramide synthases [1]. Ceramides accumulate in tissues not suited for lipid storage, mainly during conditions of metabolic dysfunction and dyslipidemia. Higher amounts of skeletal muscle ceramides have been also reported in patients with type 2 diabetes and obesity $[3,19]$. Several experimental studies suggested that distinct plasma ceramides are involved in endothelial damage and can contribute to atherosclerotic plaque erosion and, therefore, induce thrombosis [3-5,20-23]. Other studies showed that ceramides can cause a switch in the mediator of flow-induced vasodilation in coronary microcirculation from nitric oxide to hydrogen peroxide [24]. Experimental evidence in rodents also suggested that pharmacological inhibition of ceramide synthesis prevents atherogenesis [3]. On this background of experimental evidence, some prospective observational studies have recently shown that three specific plasma ceramides [i.e., $\operatorname{Cer}(\mathrm{d} 18: 1 / 16: 0), \operatorname{Cer}(\mathrm{d} 18: 1 / 18: 0)$ and $\operatorname{Cer}(\mathrm{d} 18: 1 / 24: 1)]$ predicted the development of major adverse cardiovascular events, independently of traditional cardiovascular risk factors, both in patients with stable CAD or acute coronary syndromes and in apparently healthy individuals [6-8]. Furthermore, these three plasma ceramides, and particularly Cer(d18:1/16:0), were independently associated with the presence of more vulnerable coronary plaque morphology on intravascular ultrasound virtual histology imaging in patients with established CAD undergoing coronary angiography [6]. Finally, in a prospective case-cohort study within the PREDIMED trial (Prevención con Dieta Mediterránea), enrolling nearly 1,000 elderly individuals at high cardiovascular risk but initially free from diagnosed CAD, Wang et al. showed that a Mediterranean dietary intervention had the potential to mitigate the detrimental effects of elevated levels of distinct plasma ceramides on the risk of developing major adverse cardiovascular events [9].

Overall, therefore, our findings further support the aforementioned epidemiological observations, documenting a significant association between higher levels of previously identified high-risk plasma ceramide species and the presence of inducible myocardial ischemia in patients with established or suspected CAD referred for clinically indicated stress MPS. Notably, this association was independent of traditional cardiovascular risk factors, left ventricular ejection fraction, prior history of $C A D$ and type of stress testing. 
Although the putative pathophysiological mechanisms by which specific plasma ceramides may contribute to the development of stress-induced reversible myocardial ischemia still remain elusive, we believe that this field of research is clinically relevant, and that the findings of our study could also have potential clinical implications. However, further studies are needed to better clarify the potential different biological effects of plasma ceramides with various acyl-chain lengths on signaling processes of coronary atherogenesis. Indeed, evidence has suggested that chain length-specific properties of ceramides may be implicated in different cell signaling events, and that ceramides with distinct chain lengths may be critical in several diseases [1]. Further studies are also needed to better elucidate what are the cellular sources of ceramides measured in the circulation, and what triggers the release of ceramides in patients with CAD. Recently, Ji et al. [25] reported that levels of total and very long-chain ceramides both in myocardium and in serum of patients with advanced hear failure were significantly increased compared with those in control individuals. Moreover, inhibition of de novo ceramide synthesis in rat models decreased ceramide accumulation in ischemic cardiomyopathy and reduced ventricular remodeling, fibrosis, and macrophage content following myocardial ischemia [25]. Thus, increased de novo ceramide synthesis might contribute to progressive pathologic cardiac remodeling and dysfunction. To further investigate the association between ceramide accumulation and heart function, Klevstig et al. [26] examined myocardial left ventricle biopsies from patients with chronic myocardial ischemia, and found that ceramide levels were higher in biopsies from patients with heart failure. These authors also found that although cardiac ceramide accumulation was reduced 24-hours after an induced myocardial infarction in mice with a partial deficiency in acid sphingomyelinase, this reduction was not accompanied by an improvement in heart function or survival [26].

The present study has some important limitations that should be mentioned. Firstly, the cross-sectional design of the study limits our ability to establish a cause-and-effect relationship. Secondly, patients were recruited based on their (relatively) high cardiovascular risk. Therefore, our findings might not be necessarily applicable in populations with low or intermediate cardiovascular risk. Thirdly, after adjusting for multiple comparisons, some of the $p$-values were of borderline statistical significance. However, the consistency of these results across the plasma ceramides supports the robustness of 
these observations. Although we carefully adjusted for multiple known cardiovascular risk factors, residual confounding cannot be ruled out.

Notwithstanding these limitations, our study has several major strengths, including the relatively large sample size, the consecutive enrolment of patients, the use of stress MPS for detecting inducible myocardial ischemia, and the exclusion of patients with a documented history of kidney failure, cirrhosis or cancer. We believe that inclusion of patients with these serious comorbid conditions might have confounded interpretation of the data.

In conclusion, this cross-sectional study shows for the first time that distinct plasma ceramides are independently associated with the presence of inducible myocardial ischemia in patients with established or suspected CAD undergoing stress MPS. Although further studies are required to replicate these results in other populations, our findings add insight to results of recent prospective studies supporting a significant association between distinct plasma ceramides and the long-term risk of developing adverse cardiovascular outcomes. Further studies are also needed to establish whether measurement of specific plasma ceramides in patients with established or suspected CAD could represent new therapeutic targets for the treatment and management of coronary atherosclerosis.

Conflict of Interest Disclosures: none.

Funding Sources: GT is supported in part by grants from the University School of Medicine of Verona, Verona, Italy. CDB is supported in part by grants from the Southampton National Institute for Health Research Biomedical Research Centre.

Authors' Contributions: AM, SB, MS and GT conceived and designed the study. GL measured plasma ceramides. MS performed myocardial perfusion scintigraphy. AM, SB, $\mathrm{GL}, \mathrm{CD}, \mathrm{MS}$ researched data and reviewed/edited the manuscript. CDB, SG and EB contributed to discussion and reviewed/edited the manuscript. AM and GT analyzed the data and wrote the manuscript draft. AM and GT are the guarantors of this work and, as 
such, had full access to all the data in the study and take responsibility for the integrity of the data and the accuracy of the data. All Authors approved the final version of the manuscript.

\section{REFERENCES}

1. Grösch S, Schiffmann S, Geisslinger G. Chain length-specific properties of ceramides. Prog Lipid Res 2012;51:50-62.

2. Maceyka M, Spiegel S. Sphingolipid metabolites in inflammatory disease. Nature 2014;510:58-67.

3. Meikle PJ, Summers SA. Sphingolipids and phospholipids in insulin resistance and related metabolic disorders. Nat Rev Endocrinol 2017;13:79-91.

4. Bismuth J, Lin P, Yao Q, Chen C. Ceramide: a common pathway for atherosclerosis? Atherosclerosis 2008;196:497-504.

5. Sasset L, Zhang Y, Dunn TM, Di Lorenzo A. Sphingolipid de novo biosynthesis: a rheostat of cardiovascular homeostasis. Trends Endocrinol Metab 2016;27:807-819.

6. Cheng JM, Suoniemi M, Kardys I, Vihervaara T, de Boer SP, Akkerhuis KM, SysiAho M, Ekroos K, Garcia-Garcia HM, Oemrawsingh RM, Regar E, Koenig W, Serruys PW, van Geuns RJ, Boersma E, Laaksonen R. Plasma concentrations of molecular lipid species in relation to coronary plaque characteristics and cardiovascular outcome:

Results of the ATHEROREMO-IVUS study. Atherosclerosis 2015;243:560-566.

7. Laaksonen R, Ekroos K, Sysi-Aho M, Hilvo M, Vihervaara T, Kauhanen D, Suoniemi M, Hurme R, März W, Scharnagl H, Stojakovic T, Vlachopoulou E, Lokki ML, Nieminen MS, Klingenberg R, Matter CM, Hornemann T, Jüni P, Rodondi N, Räber L, Windecker S, Gencer B, Pedersen ER, Tell GS, Nygård O, Mach F, Sinisalo J, Lüscher TF. Plasma ceramides predict cardiovascular death in patients with stable coronary artery disease and acute coronary syndromes beyond LDL-cholesterol. Eur Heart J. 2016;37:1967-1976.

8. Havulinna AS, Sysi-Aho M, Hilvo M, Kauhanen D, Hurme R, Ekroos K, Salomaa V, Laaksonen R. Circulating ceramides predict cardiovascular outcomes in the populationbased FINRISK 2002 cohort. Arterioscler Thromb Vasc Biol 2016;36:2424-2430. 
9. Wang DD, Toledo E, Hruby A, Rosner BA, Willett WC, Sun Q, Razquin C, Zheng Y, Ruiz-Canela M, Guasch-Ferré M, Corella D, Gómez-Gracia E, Fiol M, Estruch R, Ros E, Lapetra J, Fito M, Aros F, Serra-Majem L, Lee CH, Clish CB, Liang L, Salas-Salvadó J, Martínez-González MA, Hu FB. Plasma ceramides, Mediterranean diet, and incident cardiovascular disease in the PREDIMED trial (Prevención con Dieta Mediterránea). Circulation 2017;135:2028-2040.

10. Klocke FJ, Baird MG, Lorell BH, Bateman TM, Messer JV, Berman DS, O'Gara PT, Carabello BA, Russell RO Jr, Cerqueira MD, St John Sutton MG, DeMaria AN, Udelson JE, Kennedy JW, Verani MS, Williams KA, Antman EM, Smith SC Jr, Alpert JS, Gregoratos G, Anderson JL, Hiratzka LF, Faxon DP, Hunt SA, Fuster V, Jacobs AK, Gibbons RJ, Russell RO; American College of Cardiology; American Heart Association; American Society for Nuclear Cardiology. ACC/AHA/ASNC guidelines for the clinical use of cardiac radionuclide imaging--executive summary: a report of the American College of Cardiology/American Heart Association Task Force on Practice Guidelines (ACC/AHA/ASNC Committee to Revise the 1995 Guidelines for the Clinical Use of Cardiac Radionuclide Imaging). J Am Coll Cardiol 2003;42:1318-1333.

11. Levey AS, Bosch JP, Lewis JB, Greene T, Rogers N, Roth D; Modification of Diet in Renal Disease Study Group. A more accurate method to estimate glomerular filtration rate from serum creatinine: a new prediction equation. Ann Intern Med 1999;130:461470.

12. Kauhanen D, Sysi-Aho M, Koistinen KM, Laaksonen R, Sinisalo J, Ekroos K. Development and validation of a high-throughput LC-MS/MS assay for routine measurement of molecular ceramides. Anal Bioanal Chem 2016;408:3475-3483. 13. Bonapace S, Valbusa F, Bertolini L, Zenari L, Canali G, Molon G, Lanzoni L, Cecchetto A, Rossi A, Mantovani A, Zoppini G, Barbieri E, Targher G. Early impairment in left ventricular longitudinal systolic function is associated with an increased risk of incident atrial fibrillation in patients with type 2 diabetes. J Diabetes Complications 2017;31:413-418.

14. Roffi M, Patrono C, Collet JP, Mueller C, Valgimigli M, Andreotti F, Bax JJ, Borger MA, Brotons C, Chew DP, Gencer B, Hasenfuss G, Kjeldsen K, Lancellotti P, Landmesser U, Mehilli J, Mukherjee D, Storey RF, Windecker S, Baumgartner H, Gaemperli O, Achenbach S, Agewall S, Badimon L, Baigent C, Bueno H, Bugiardini R, Carerj S, Casselman F, Cuisset T, Erol Ç, Fitzsimons D, Halle M, Hamm C, HildickSmith D, Huber K, Iliodromitis E, James S, Lewis BS, Lip GY, Piepoli MF, Richter D, 
Rosemann T2, Sechtem U, Steg PG, Vrints C, Luis Zamorano J; Management of Acute Coronary Syndromes in Patients Presenting without Persistent ST-Segment Elevation of the European Society of Cardiology. 2015 ESC Guidelines for the management of acute coronary syndromes in patients presenting without persistent ST-segment elevation: Task Force for the management of acute coronary syndromes in patients presenting without persistent ST-segment elevation of the European Society of Cardiology (ESC). Eur Heart J 2016;37:267-315.

15. Tarasov K, Ekroos K, Suoniemi M, Kauhanen D, Sylvanne T, Hurme R, GouniBerthold I, Berthold HK, Kleber ME, Laaksonen R, Marz W. Molecular lipids identify cardiovascular risk and are efficiently lowered by simvastatin and PCSK9 deficiency. J Clin Endocrinol Metab 2014;99:E45-E52.

16. Meikle PJ, Wong G, Tsorotes D, Barlow CK, Weir JM, Christopher MJ, Maclntosh GL, Goudey B, Stern L, Kowalczyk A, Haviv I, White AJ, Dart AM, Duffy SJ, Jennings GL, Kingwell BA, Weir M. Plasma lipidomic analysis of stable and unstable coronary artery disease. Arterioscler Thromb Vasc Biol 2011;31:2723-2732.

17. Fernandez C, Sandin M, Sampaio JL, Almgren P, Narkiewicz K, Hoffmann M, Hedner T, Wahlstrand B, Simons K, Shevchenko A, James P, Melander O. Plasma lipid composition and risk of developing cardiovascular disease. PLoS One 2013;8:e71846.

18. Chatterjee M, Rath $D$, Schlotterbeck J, Rheinlaender J, Walker-Allgaier B, Alnaggar N, Zdanyte M, Müller I, Borst O, Geisler T, Schäffer TE, Lämmerhofer M, Gawaz M. Regulation of oxidized platelet lipidome: implications for coronary artery disease. Eur Heart J 2017;38:1993-2005.

19. Broskey NT, Obanda DN, Burton JH, Cefalu WT, Ravussin E. Skeletal muscle ceramides and daily fat oxidation in obesity and diabetes. Metabolism 2018 Jan 4. pii: S0026-0495(17)30359-1. doi: 10.1016/j.metabol.2017.12.012 [Epub ahead of print]. 20. Halasiddappa LM, Koefeler H, Futerman AH, Hermetter A. Oxidized phospholipids induce ceramide accumulation in RAW 264.7 macrophages: role of ceramide synthases. PLoS One 2013;8:e70002.

21. Syed I, Jayaram B, Subasinghe W, Kowluru A. Tiam1/Rac1 signaling pathway mediates palmitate-induced, ceramide-sensitive generation of superoxides and lipid peroxides and the loss of mitochondrial membrane potential in pancreatic beta-cells. Biochem Pharmacol 2010;80:874-883.

22. Law BA, Liao X, Moore KS, Southard A, Roddy P, Ji R, Sculz Z, Bielawska A, Schulze PC, Cowart LA. Lipotoxic very-long-chain ceramides cause mitochondrial 
dysfunction, oxidative stress, and cell death in cardiomyocytes. FASEB J 2018;32:14031416.

23. Freed JK, Beyer AM, LoGiudice JA, Hockenberry JC, Gutterman DD. Ceramide changes the mediator of flow-induced vasodilation from nitric oxide to hydrogen peroxide in the human microcirculation. Circ Res 2014;115: 525-532.

24. Park JW, Park WJ, Futerman AH. Ceramide synthases as potential targets for therapeutic intervention in human diseases. Biochim Biophys Acta 2014;1841:671-681. 25. Ji R, Akashi H, Drosatos K, Liao X, Jiang H, Kennel PJ, Brunjes DL, Castillero E, Zhang X, Deng LY, Homma S, George IJ, Takayama H, Naka Y, Goldberg IJ, Schulze $\mathrm{PC}$. Increased de novo ceramide synthesis and accumulation in failing myocardium. JCI Insight 2017;2(9).pii: 82922.

26. Klevstig $M$, Ståhlman $M$, Lundqvist $A$, Scharin Täng $M$, Fogelstrand $P$, Adiels $M$, Andersson L, Kolesnick R, Jeppsson A, Borén J, Levin MC. Targeting acid sphingomyelinase reduces cardiac ceramide accumulation in the post-ischemic heart. $J$ Mol Cell Cardiol 2016;93:69-72. 
Table 1. Main clinical and biochemical characteristics in patients stratified by either absence or presence of inducible myocardial perfusion defects on stress myocardial perfusion scintigraphy.

\begin{tabular}{|c|c|c|c|}
\hline & $\begin{array}{l}\text { Without defects } \\
(n=89)\end{array}$ & $\begin{array}{l}\text { With defects } \\
(n=78)\end{array}$ & $\begin{array}{l}P \\
\text { value }\end{array}$ \\
\hline Age (years) & $68.8 \pm 10$ & $71.8 \pm 9$ & $<0.05$ \\
\hline Sex (men) $(\%)$ & 60.7 & 96.2 & $<0.001$ \\
\hline Body mass index $\left(\mathrm{kg} / \mathrm{m}^{2}\right)$ & $26.8 \pm 4.1$ & $27.7 \pm 4.0$ & 0.23 \\
\hline Systolic blood pressure $(\mathrm{mmHg})$ & $138 \pm 18$ & $132 \pm 15$ & 0.11 \\
\hline Diastolic blood pressure $(\mathrm{mmHg})$ & $78 \pm 9$ & $76 \pm 8$ & 0.20 \\
\hline Total cholesterol (mmol/L) & $4.4 \pm 1.0$ & $4.1 \pm 1.1$ & 0.25 \\
\hline LDL-cholesterol (mmol/L) & $2.7 \pm 0.9$ & $2.4 \pm 1.1$ & 0.09 \\
\hline HDL-cholesterol (mmol/L) & $1.3 \pm 0.3$ & $1.2 \pm 0.3$ & 0.74 \\
\hline Triglycerides (mmol/L) & $1.5 \pm 0.8$ & $1.4 \pm 0.6$ & 0.64 \\
\hline Fasting glucose (mmol/L), $n=82$ & $6.1 \pm 1.7$ & $6.3 \pm 1.8$ & 0.77 \\
\hline $\mathrm{HbA} 1 \mathrm{c}(\mathrm{mmol} / \mathrm{mol}), \mathrm{n}=40$ & $50.2 \pm 7$ & $46.6 \pm 4$ & 0.63 \\
\hline e-GFR ${ }_{M D R D}\left(\mathrm{~mL} / \mathrm{min} / 1.73 \mathrm{~m}^{2}\right)$ & $78 \pm 20$ & $79 \pm 24$ & 0.72 \\
\hline LV ejection fraction (\%) & $62.1 \pm 10$ & $49.5 \pm 11$ & $<0.001$ \\
\hline Chronic kidney disease (\%) & 6.7 & 14.1 & 0.12 \\
\hline Current smokers (\%) & 6.8 & 16.7 & 0.14 \\
\hline Dyslipidemia (\%) & 92.1 & 93.6 & 0.72 \\
\hline Coronary artery disease (\%) & 39.1 & 74.4 & $<0.001$ \\
\hline Hypertension (\%) & 73.6 & 87.2 & $<0.05$ \\
\hline Diabetes (\%) & 18.4 & 30.8 & 0.06 \\
\hline Valvular heart disease (\%) & 9.2 & 6.4 & 0.51 \\
\hline Atrial fibrillation (\%) & 5.8 & 14.1 & 0.07 \\
\hline Chronic obstructive pulmonary disease (\%) & 6.9 & 6.4 & 0.90 \\
\hline Stroke (\%) & 1.1 & 6.4 & 0.08 \\
\hline Antiplatelet drug users (\%) & 57.5 & 71.8 & $<0.05$ \\
\hline Anticoagulant drug users (\%) & 5.8 & 25.6 & $<0.001$ \\
\hline Digitalis (\%) & 1.1 & 1.3 & 0.94 \\
\hline Antiarrhythmic drug users (\%) & 6.9 & 5.1 & 0.64 \\
\hline Beta-blocker users (\%) & 37.9 & 58.9 & $<0.01$ \\
\hline ACE-I/ARB users (\%) & 56.3 & 66.7 & 0.17 \\
\hline Calcium-channel blocker users (\%) & 11.5 & 10.3 & 0.80 \\
\hline Diuretic users (\%) & 22.9 & 34.6 & 0.10 \\
\hline Nitrate users (\%) & 9.2 & 24.4 & $<0.01$ \\
\hline
\end{tabular}




\begin{tabular}{llll}
\hline Statin users (\%) & 50.6 & 74.4 & $<0.01$ \\
Insulin users (\%), n=40 & 2.3 & 5.1 & 0.33 \\
Oral hypoglycemic drug users (\%), n=40 & 14.9 & 23.1 & 0.18 \\
\hline
\end{tabular}

\begin{abstract}
Sample size, $n=167$ unless otherwise indicated. Data are expressed as means \pm SD or percentages. Differences between the two groups were tested by the chi-squared test for categorical variables, the unpaired Student's $t$-test for normally

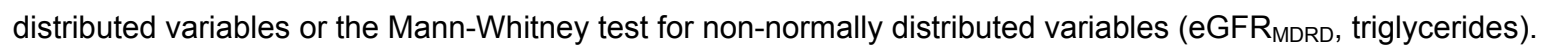

Note: Presence of chronic kidney disease was defined as e-GFR $\mathrm{MDRD}_{2}<60 \mathrm{ml} / \mathrm{min} / 1.73 \mathrm{~m}^{2}$; dyslipidemia was defined as total cholesterol $\geq 5.2 \mathrm{mmol} / \mathrm{L}$ ( $\geq 200 \mathrm{mg} / \mathrm{dL}$ ) and/or use of any lipid-lowering agents; hypertension was defined as blood pressure $\geq 140 / 90 \mathrm{mmHg}$ and/or use of any anti-hypertensive agents. HbA1c levels and information on use of glucoselowering drugs were available only for patients with established diabetes $(n=40)$.

Abbreviations: ACE-I, angiotensin-converting-enzyme inhibitor; ARB, angiotensin II receptor blocker; e-GFR $\mathrm{MDRD}_{\text {, }}$ glomerular filtration rate estimated by using the Modification of Diet in Renal Diseases (MDRD) study equation; LV, left ventricular. 
Table 2. Main clinical and biochemical characteristics in patients stratified by type of stress on myocardial perfusion scintigraphy.

\begin{tabular}{|c|c|c|c|}
\hline & $\begin{array}{l}\text { Exercise group } \\
(n=94)\end{array}$ & $\begin{array}{l}\text { Dipyridamole group } \\
(n=73)\end{array}$ & $\begin{array}{l}P \\
\text { value }\end{array}$ \\
\hline Age (years) & $66.8 \pm 10$ & $74.8 \pm 7$ & $<0.05$ \\
\hline Sex (men) $(\%)$ & 80.5 & 72.6 & 0.21 \\
\hline Body mass index $\left(\mathrm{kg} / \mathrm{m}^{2}\right)$ & $27.0 \pm 4.1$ & $27.8 \pm 4.2$ & 0.38 \\
\hline Systolic blood pressure $(\mathrm{mmHg})$ & $135 \pm 15$ & $143 \pm 19$ & $<0.05$ \\
\hline Diastolic blood pressure $(\mathrm{mmHg})$ & $81 \pm 9$ & $83 \pm 9$ & 0.31 \\
\hline Total cholesterol (mmol/L) & $4.3 \pm 1.0$ & $4.2 \pm 1.1$ & 0.87 \\
\hline LDL-cholesterol (mmol/L) & $2.5 \pm 1.0$ & $2.5 \pm 1.2$ & 0.90 \\
\hline HDL-cholesterol (mmol/L) & $1.2 \pm 0.4$ & $1.3 \pm 0.2$ & 0.52 \\
\hline Triglycerides (mmol/L) & $1.4 \pm 0.6$ & $1.5 \pm 0.7$ & 0.41 \\
\hline Fasting glucose (mmol/L), $\mathrm{n}=82$ & $6.0 \pm 1.7$ & $7.1 \pm 3.6$ & 0.07 \\
\hline $\mathrm{HbA} 1 \mathrm{c}(\mathrm{mmol} / \mathrm{mol}), \mathrm{n}=40$ & $42.8 \pm 8$ & $52.3 \pm 9$ & 0.21 \\
\hline e-GFR ${ }_{M D R D}\left(\mathrm{~mL} / \mathrm{min} / 1.73 \mathrm{~m}^{2}\right)$ & $82 \pm 21$ & $75 \pm 24$ & 0.13 \\
\hline LV ejection fraction (\%) & $57.3 \pm 12$ & $52.8 \pm 15$ & $=0.05$ \\
\hline Chronic kidney disease (\%) & 8.5 & 12.3 & 0.42 \\
\hline Current smokers (\%) & 10.6 & 5.4 & 0.41 \\
\hline Dyslipidemia (\%) & 91.5 & 94.5 & 0.45 \\
\hline Coronary artery disease (\%) & 51.1 & 61.6 & 0.17 \\
\hline Hypertension (\%) & 73.9 & 86.7 & $<0.05$ \\
\hline Diabetes (\%) & 17.2 & 33.3 & $<0.05$ \\
\hline Valvular heart disease (\%) & 8.7 & 6.8 & 0.66 \\
\hline Atrial fibrillation (\%) & 6.5 & 13.7 & 0.12 \\
\hline Chronic obstructive pulmonary disease (\%) & 8.7 & 4.1 & 0.24 \\
\hline Stroke $(\%)$ & 3.3 & 4.1 & 0.78 \\
\hline Antiplatelet drug users (\%) & 69.6 & 57.5 & 0.11 \\
\hline Anticoagulant drug users (\%) & 8.7 & 23.3 & $<0.05$ \\
\hline Digitalis (\%) & 2.0 & 0.0 & 0.20 \\
\hline Antiarrhythmic drug users (\%) & 5.4 & 6.8 & 0.71 \\
\hline Beta-blocker users (\%) & 45.6 & 50.7 & 0.52 \\
\hline ACE-I/ARB users (\%) & 57.6 & 65.8 & 0.29 \\
\hline Calcium-channel blocker users (\%) & 11.5 & 10.3 & 0.80 \\
\hline Diuretic users (\%) & 19.6 & 39.7 & $<0.05$ \\
\hline Nitrate users (\%) & 17.4 & 15.1 & 0.69 \\
\hline Statin users (\%) & 59.8 & 64.4 & 0.87 \\
\hline
\end{tabular}




\begin{tabular}{llll}
\hline Insulin users (\%), $\mathrm{n}=40$ & 1.1 & 6.8 & $<0.05$ \\
Oral hypoglycemic drug users (\%), $\mathrm{n}=40$ & 13.0 & 26.0 & $<0.05$
\end{tabular}

Sample size, $n=167$ unless otherwise indicated. Data are expressed as means \pm SD or percentages. Differences between the two groups were tested by the chi-squared test for categorical variables, the unpaired Student's $t$-test for normally

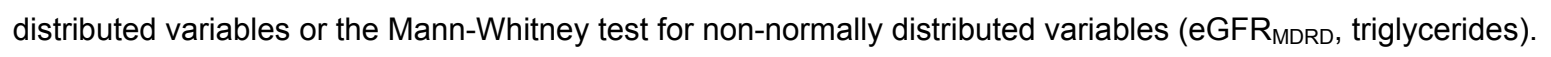

Note: Presence of chronic kidney disease was defined as e-GFR $\mathrm{MDRD}_{2}<60 \mathrm{ml} / \mathrm{min} / 1.73 \mathrm{~m}^{2}$; dyslipidemia was defined as total cholesterol $\geq 5.2 \mathrm{mmol} / \mathrm{L}$ ( $\geq 200 \mathrm{mg} / \mathrm{dL}$ ) and/or use of any lipid-lowering agents; hypertension was defined as blood pressure $\geq 140 / 90 \mathrm{mmHg}$ and/or use of any anti-hypertensive agents. HbA1c levels and information on current use of glucose-lowering drugs were available only for patients with established diabetes $(n=40)$.

Abbreviations: ACE-I, angiotensin-converting-enzyme inhibitor; ARB, angiotensin II receptor blocker; e-GFR $\mathrm{RDRD}_{\text {, }}$ glomerular filtration rate estimated by using the Modification of Diet in Renal Diseases (MDRD) study equation; LV, left ventricular. 
Table 3. Basal and post-stress plasma ceramide levels [and their ratios to Cer(d18:1/24:0)] in patients stratified by absence or presence of inducible myocardial perfusion defects on stress myocardial perfusion scintigraphy.

\begin{tabular}{|c|c|c|c|}
\hline & $\begin{array}{l}\text { Without defects } \\
(n=89)\end{array}$ & $\begin{array}{l}\text { With defects } \\
(n=78)\end{array}$ & $\begin{array}{l}P \\
\text { value* }\end{array}$ \\
\hline \multicolumn{4}{|l|}{ Basal } \\
\hline Cer(d18:1/16:0) (umol/L) & $0.326 \pm 0.08$ & $0.321 \pm 0.08$ & 0.36 \\
\hline Cer(d18:1/18:0) (umol/L) & $0.138 \pm 0.06$ & $0.148 \pm 0.07$ & 0.07 \\
\hline Cer(d18:1/20:0) (umol/L) & $0.101 \pm 0.03$ & $0.111 \pm 0.04$ & $<0.05$ \\
\hline Cer(d18:1/22:0) (umol/L) & $0.707 \pm 0.26$ & $0.742 \pm 0.26$ & $<0.05$ \\
\hline Cer(d18:1/24:0) (umol/L) & $2.849 \pm 0.77$ & $2.789 \pm 0.80$ & 0.59 \\
\hline Cer(d18:1/24:1) (umol/L) & $1.082 \pm 0.38$ & $1.245 \pm 0.47$ & $<0.05$ \\
\hline Cer(d18:1/16:0)/Cer(d18:1/24:0) & $0.119 \pm 0.03$ & $0.120 \pm 0.03$ & 0.96 \\
\hline Cer(d18:1/18:0)/Cer(d18:1/24:0) & $0.049 \pm 0.02$ & $0.054 \pm 0.02$ & 0.26 \\
\hline Cer(d18:1/20:0)/Cer(d18:1/24:0) & $0.056 \pm 0.03$ & $0.064 \pm 0.03$ & 0.24 \\
\hline $\operatorname{Cer}(\mathrm{d} 18: 1 / 22: 0) / \operatorname{Cer}(\mathrm{d} 18: 1 / 24: 0)$ & $0.250 \pm 0.06$ & $0.267 \pm 0.06$ & $<0.05$ \\
\hline $\operatorname{Cer}(\mathrm{d} 18: 1 / 24: 1) / \operatorname{Cer}(\mathrm{d} 18: 1 / 24: 0)$ & $0.396 \pm 0.14$ & $0.456 \pm 0.14$ & $<0.05$ \\
\hline \multicolumn{4}{|l|}{ Post-stress } \\
\hline Cer(d18:1/16:0) (umol/L) & $0.332 \pm 0.08$ & $0.324 \pm 0.08$ & 0.45 \\
\hline Cer(d18:1/18:0) (umol/L) & $0.142 \pm 0.07$ & $0.154 \pm 0.08$ & 0.09 \\
\hline Cer(d18:1/20:0) (umol/L) & $0.105 \pm 0.04$ & $0.111 \pm 0.05$ & 0.20 \\
\hline Cer(d18:1/22:0) (umol/L) & $0.718 \pm 0.27$ & $0.743 \pm 0.26$ & 0.06 \\
\hline Cer(d18:1/24:0) (umol/L) & $2.891 \pm 0.78$ & $2.822 \pm 0.83$ & 0.61 \\
\hline Cer(d18:1/24:1) (umol/L) & $1.118 \pm 0.40$ & $1.285 \pm 0.47$ & $<0.05$ \\
\hline $\operatorname{Cer}(\mathrm{d} 18: 1 / 16: 0) / \operatorname{Cer}(\mathrm{d} 18: 1 / 24: 0)$ & $0.120 \pm 0.03$ & $0.119 \pm 0.03$ & 0.96 \\
\hline $\operatorname{Cer}(\mathrm{d} 18: 1 / 18: 0) / \operatorname{Cer}(\mathrm{d} 18: 1 / 24: 0)$ & $0.051 \pm 0.02$ & $0.055 \pm 0.02$ & 0.28 \\
\hline $\operatorname{Cer}(\mathrm{d} 18: 1 / 20: 0) / \operatorname{Cer}(\mathrm{d} 18: 1 / 24: 0)$ & $0.057 \pm 0.03$ & $0.062 \pm 0.03$ & 0.49 \\
\hline Cer(d18:1/22:0)/Cer(d18:1/24:0) & $0.249 \pm 0.06$ & $0.265 \pm 0.06$ & $<0.05$ \\
\hline $\operatorname{Cer}(d 18: 1 / 24: 1) / \operatorname{Cer}(d 18: 1 / 24: 0)$ & $0.404 \pm 0.15$ & $0.468 \pm 0.14$ & $<0.05$ \\
\hline
\end{tabular}

Sample size, $n=167$. Data are expressed as means \pm SD. Differences between patients with and those without stressinduced myocardial ischemia were tested by the Mann-Whitney $U$ test. * $P$-values adjusted for age and sex. 
Table 4. Association between basal plasma ceramides [and their ratio to $\operatorname{Cer}(\mathrm{d} 18: 1 / 24: 0)$ ] and presence of inducible myocardial perfusion defects on stress myocardial perfusion scintigraphy.

\begin{tabular}{|c|c|c|c|}
\hline Logistic Regression Models & Odds ratio & $95 \% \mathrm{Cl}$ & $P$ value \\
\hline \multicolumn{4}{|c|}{ 1-SD increment (i.e., +0.08 umol/L) in $\operatorname{Cer(d18:1/16:0)}$} \\
\hline Unadjusted model & 0.95 & $0.69-1.29$ & 0.72 \\
\hline Adjusted model 1 & 1.23 & $0.84-1.79$ & 0.29 \\
\hline Adjusted model 2 & 1.61 & $1.02-2.67$ & $<0.05$ \\
\hline \multicolumn{4}{|c|}{ 1-SD increment (+0.07 umol/L) in $\operatorname{Cer}(\mathrm{d} 18: 1 / 18: 0)$} \\
\hline Unadjusted model & 1.18 & $0.87-1.61$ & 0.28 \\
\hline Adjusted model 1 & 1.46 & $0.99-2.18$ & $=0.05$ \\
\hline Adjusted model 2 & 1.71 & $1.04-2.83$ & $<0.05$ \\
\hline \multicolumn{4}{|c|}{ 1-SD increment (+0.04 umol/L) in $\operatorname{Cer}(\mathrm{d} 18: 1 / 20: 0)$} \\
\hline Unadjusted model & 1.29 & $0.95-1.77$ & 0.11 \\
\hline Adjusted model 1 & 1.52 & $1.03-2.24$ & $<0.05$ \\
\hline Adjusted model 2 & 1.95 & $1.15-3.31$ & $<0.01$ \\
\hline \multicolumn{4}{|c|}{ 1-SD increment (+0.26 umol/L) in $\operatorname{Cer}(\mathrm{d} 18: 1 / 22: 0)$} \\
\hline Unadjusted model & 1.14 & $0.84-1.55$ & 0.39 \\
\hline Adjusted model 1 & 1.49 & $1.02-2.19$ & $<0.05$ \\
\hline Adjusted model 2 & 1.91 & $1.12-3.25$ & $<0.01$ \\
\hline \multicolumn{4}{|c|}{ 1-SD increment (+0.43 umol/L) in $\operatorname{Cer}(d 18: 1 / 24: 1)$} \\
\hline Unadjusted model & 1.48 & $1.08-2.04$ & $<0.01$ \\
\hline Adjusted model 1 & 1.56 & $1.08-2.28$ & $<0.01$ \\
\hline Adjusted model 2 & 2.09 & $1.24-3.57$ & $<0.01$ \\
\hline \multicolumn{4}{|c|}{ 1-SD increment (+0.78 umol/L) in $\operatorname{Cer}(\mathrm{d} 18: 1 / 24: 0)$} \\
\hline Unadjusted model & 0.92 & $0.68-1.26$ & 0.62 \\
\hline Adjusted model 1 & 1.12 & $0.78-1.59$ & 0.54 \\
\hline Adjusted model 2 & 1.55 & $0.98-2.18$ & 0.06 \\
\hline \multicolumn{4}{|c|}{ 1-SD increment (+0.03) in Cer(d18:1/16:0)/Cer(d18:1/24:0) } \\
\hline Unadjusted model & 1.00 & $0.74-1.36$ & 0.97 \\
\hline Adjusted model 1 & 0.99 & $0.69-1.08$ & 0.98 \\
\hline Adjusted model 2 & 0.96 & $0.58-1.44$ & 0.66 \\
\hline \multicolumn{4}{|c|}{ 1-SD increment (+0.02) in Cer(d18:1/18:0)/Cer(d18:1/24:0) } \\
\hline Unadjusted model & 1.18 & $0.86-1.60$ & 0.30 \\
\hline Adjusted model 1 & 1.28 & $0.87-1.87$ & 0.21 \\
\hline Adjusted model 2 & 1.24 & $0.79-1.94$ & 0.35 \\
\hline \multicolumn{4}{|c|}{ 1-SD increment $(+0.03)$ in $\operatorname{Cer}(d 18: 1 / 20: 0) / \operatorname{Cer}(d 18: 1 / 24: 0)$} \\
\hline
\end{tabular}




\begin{tabular}{lccl}
\hline Unadjusted model & 1.28 & $0.94-1.75$ & 0.97 \\
Adjusted model 1 & 1.46 & $0.97-1.84$ & 0.26 \\
Adjusted model 2 & 1.62 & $1.00-2.62$ & $=0.05$ \\
1-SD increment (+0.06) in Cer(d18:1/22:0)/Cer(d18:1/24:0) & \\
Unadjusted model & 1.35 & $0.98-1.85$ & 0.06 \\
Adjusted model 1 & 1.49 & $1.03-2.15$ & $<0.05$ \\
Adjusted model 2 & 1.38 & $1.01-2.22$ & $=0.05$ \\
1-SD increment (+0.15) in Cer(d18:1/24:1)/Cer(d18:1/24:0) & \\
Unadjusted model & 1.54 & $1.11-2.13$ & $<0.05$ \\
Adjusted model 1 & 1.36 & $0.93-1.99$ & 0.10 \\
Adjusted model 2 & 1.41 & $1.02-2.30$ & $<0.05$
\end{tabular}

\footnotetext{
Sample size, $n=167$. Data are expressed as odds ratios $\pm 95 \%$ confidence intervals $(\mathrm{Cl})$ as assessed by either univariate (unadjusted) or multivariate logistic regression analysis. The presence of inducible myocardial perfusion defects on stress MPS was included as the dependent variable. Each ceramide as well as its ratios to Cer(d18:1/24:0) were expressed per 1-SD increment.
}

Other covariates included in these multivariate logistic regression models (along with distinct plasma ceramides) were as follows: model 1: adjusted for age and sex; model 2: adjusted for age, sex, diabetes, hypertension (blood pressure $\geq 140 / 90 \mathrm{mmHg}$ and/or drug treatment), dyslipidemia (total cholesterol $\geq 5.2 \mathrm{mmol} / \mathrm{L}$ and/or drug treatment), smoking, prior history of CAD, LV ejection fraction, and type of MPS stress testing (exercise vs. dipyridamole). 
Table 5. Association between post-stress plasma ceramides [and their ratio to Cer(d18:1/24:0)] and presence of inducible myocardial perfusion defects on stress myocardial perfusion scintigraphy.

\begin{tabular}{|c|c|c|c|}
\hline Logistic Regression Models & Odds ratio & $95 \% \mathrm{Cl}$ & $P$ value \\
\hline \multicolumn{4}{|c|}{ 1-SD increment (i.e. +0.08 umol/L) in Cer(d18:1/16:0) } \\
\hline Unadjusted model & 0.91 & $0.67-1.24$ & 0.54 \\
\hline Adjusted model 1 & 1.18 & $0.81-1.71$ & 0.39 \\
\hline Adjusted model 2 & 1.88 & $1.10-3.21$ & $<0.05$ \\
\hline \multicolumn{4}{|c|}{ 1-SD increment (+0.07 umol/L) in $\operatorname{Cer}(\mathrm{d} 18: 1 / 18: 0)$} \\
\hline Unadjusted model & 1.17 & $0.86-1.59$ & 0.32 \\
\hline Adjusted model 1 & 1.42 & $0.96-2.13$ & 0.07 \\
\hline Adjusted model 2 & 1.82 & $1.09-3.01$ & $<0.05$ \\
\hline \multicolumn{4}{|c|}{ 1-SD increment (+0.04 umol/L) in $\operatorname{Cer}(\mathrm{d} 18: 1 / 20: 0)$} \\
\hline Unadjusted model & 1.15 & $0.85-1.57$ & 0.36 \\
\hline Adjusted model 1 & 1.29 & $0.90-1.87$ & 0.18 \\
\hline Adjusted model 2 & 1.75 & $1.05-2.90$ & $<0.05$ \\
\hline \multicolumn{4}{|c|}{ 1-SD increment (+0.27 umol/L) in Cer(d18:1/22:0) } \\
\hline Unadjusted model & 1.10 & $0.81-1.49$ & 0.54 \\
\hline Adjusted model 1 & 1.46 & $1.00-2.14$ & $<0.05$ \\
\hline Adjusted model 2 & 2.23 & $1.24-4.02$ & $<0.01$ \\
\hline \multicolumn{4}{|c|}{ 1-SD increment (+0.44 umol/L) in Cer(d18:1/24:1) } \\
\hline Unadjusted model & 1.49 & $1.08-2.05$ & $<0.01$ \\
\hline Adjusted model 1 & 1.57 & $1.07-2.29$ & $<0.01$ \\
\hline Adjusted model 2 & 2.44 & $1.41-4.24$ & $<0.01$ \\
\hline \multicolumn{4}{|c|}{ 1-SD increment $(+0.80$ umol/L) in $\operatorname{Cer}(\mathrm{d} 18: 1 / 24: 0)$} \\
\hline Unadjusted model & 0.92 & $0.67-1.24$ & 0.58 \\
\hline Adjusted model 1 & 1.11 & $0.79-1.58$ & 0.53 \\
\hline Adjusted model 2 & 1.61 & $0.99-2.63$ & $=0.053$ \\
\hline \multicolumn{4}{|c|}{ 1-SD increment $(+0.03)$ in $\operatorname{Cer}(\mathrm{d} 18: 1 / 16: 0) / \operatorname{Cer}(\mathrm{d} 18: 1 / 24: 0)$} \\
\hline Unadjusted model & 0.99 & $0.73-1.34$ & 0.94 \\
\hline Adjusted model 1 & 0.99 & $0.69-1.43$ & 0.99 \\
\hline Adjusted model 2 & 1.00 & $0.64-1.58$ & 0.98 \\
\hline \multicolumn{4}{|c|}{ 1-SD increment $(+0.02)$ in $\operatorname{Cer}(d 18: 1 / 18: 0) / \operatorname{Cer}(d 18: 1 / 24: 0)$} \\
\hline Unadjusted model & 1.17 & $0.86-1.59$ & 0.31 \\
\hline Adjusted model 1 & 1.27 & $0.86-1.86$ & 0.22 \\
\hline Adjusted model 2 & 1.28 & $0.81-1.99$ & 0.29 \\
\hline
\end{tabular}




\begin{tabular}{lccc}
\hline 1-SD increment (+0.03) in Cer(d18:1/20:0)/Cer(d18:1/24:0) & \\
Unadjusted model & 1.19 & $0.87-1.62$ & 0.27 \\
Adjusted model 1 & 1.14 & $0.79-1.65$ & 0.48 \\
Adjusted model 2 & 1.53 & $0.98-2.45$ & 0.06 \\
1-SD increment (+0.06) in Cer(d18:1/22:0)/Cer(d18:1/24:0) & \\
Unadjusted model & 1.31 & $0.96-1.79$ & 0.09 \\
Adjusted model 1 & 1.48 & $1.02-2.16$ & $<0.05$ \\
Adjusted model 2 & 1.54 & $1.01-2.37$ & $<0.05$ \\
1-SD increment (+0.15) in Cer(d18:1/24:1)/Cer(d18:1/24:0) & \\
Unadjusted model & 1.58 & $1.14-2.18$ & $<0.05$ \\
Adjusted model 1 & 1.41 & $0.98-2.06$ & 0.06 \\
Adjusted model 2 & 1.56 & $1.02-2.58$ & $<0.05$ \\
\hline
\end{tabular}

Sample size, $n=167$. Data are expressed as odds ratios $\pm 95 \%$ confidence intervals $(\mathrm{Cl})$ as assessed by either univariate (unadjusted) or multivariate logistic regression analysis. The presence of inducible myocardial perfusion defects on stress MPS was included as the dependent variable. Each ceramide as well as its ratios to Cer(d18:1/24:0) were expressed per 1-SD increment.

Other covariates included in these multivariate logistic regression models (along with distinct plasma ceramides) were as follows: model 1: adjusted for age and sex; model 2: adjusted for age, sex, diabetes, hypertension (blood pressure $\geq 140 / 90 \mathrm{mmHg}$ and/or drug treatment), dyslipidemia (total cholesterol $\geq 5.2 \mathrm{mmol} / \mathrm{L}$ and/or drug treatment), smoking, prior history of CAD, LV ejection fraction, and type of MPS stress testing (exercise vs. dipyridamole). 


\section{FIGURE LEGENDS}

Figure 1. Percentages of patients, stratified by presence or absence of stress-induced myocardial perfusion defects, who responded with either an increase (arbitrarily defined as an increment of at least $10 \%$ over the basal), decrease $(-10 \%$ of basal) or no changes (from $0 \%$ to $+/-9.9 \%$ of basal) in the measured plasma ceramide levels after stress MPS.

Differences between patients with and without inducible myocardial perfusion defects were tested by the chi-squared test. 
